\title{
Scolia
}

Revue de linguistique

$32 \mid 2018$

Autour des pseudos-clivées

\section{Examen d'une famille de constructions : les constructions identificatives}

Denis Apothéloz

\section{OpenEdition}

\section{Journals}

Édition électronique

URL : http://journals.openedition.org/scolia/284

DOI : $10.4000 /$ scolia.284

ISSN : 2677-4224

Éditeur

Presses universitaires de Strasbourg

Édition imprimée

Date de publication : 3 juillet 2018

Pagination : 13-41

ISBN : 979-10-344-0021-8

ISSN : $1253-9708$

Référence électronique

Denis Apothéloz, "Examen d'une famille de constructions : les constructions identificatives », Scolia [En ligne], 32 | 2018, mis en ligne le 09 avril 2019, consulté le 18 mai 2019. URL : http:// journals.openedition.org/scolia/284; DOI : 10.4000/scolia.284 
SCOLIA 32 / 2018, p. 13-41.

\title{
Examen d'une famille de constructions: les constructions identificatives ${ }^{1}$
}

\author{
Denis ApothéLOz \\ Université de Lorraine, ATILF (UMR 7118) \\ denis.apotheloz@univ-lorraine.fr
}

\section{Introduction : pseudo-clivées et constructions identificatives}

Les avis des linguistes divergent sur la façon de définir, et donc de délimiter, le champ des pseudo-clivées. Deux points toutefois paraissent faire à peu près l'unanimité:

- le premier est que les pseudo-clivées ont une forme dans laquelle on peut distinguer trois constituants: un constituant gauche, une copule et un constituant droit, soit: «A c'est $\mathrm{B} »$;

- le second point concerne leur dynamique informationnelle: le constituant gauche des pseudo-clivées est marqué comme topical (ou thématique) et comporte une présupposition. Pour cette raison, Halliday (1985) appelle ces constructions «thematic equatives» et les rattache à la famille des «identifying clauses». Il les décrit comme suit:

1 Je remercie les deux relecteurs anonymes pour la qualité et la sagacité de leurs remarques. Celles concernant la catégorisation proposée dans la section 2 de l'article, maintenant remaniée, étaient particulièrement pertinentes. Il va de soi que j'assume l'entière responsabilité pour les erreurs ou maladresses qui pourraient subsister. 
In a thematic equative, all the elements are organized into two constituents; these two are then linked by a relationship of identity, a kind of 'equal sign', expressed by some form of the verb be. [...] A thematic equative (which is sometimes called a 'pseudo-cleft sentence' in formal grammar) is simply an identifying clause with a thematic nominalization in it. (Halliday, 1985: 41, 43)

C'est tout d'abord le lien entre les constituants gauche et droit que je voudrais examiner, ce qui revient à se pencher sur la nature de la prédication marquée par la copule. Comme le fait Halliday, j'utiliserai le terme d' «identification" pour désigner cette prédication (plus souvent nommée «spécification» dans la littérature, appellation dont la paternité est attribuée à Akmajian (1970)), et je désignerai cette famille de constructions par l'expression de "constructions identificatives $»^{2}$.

La notion de construction identificative se veut donc plus générale que celle de pseudo-clivée. Elle vise à englober un grand nombre de formes qui ont toutes en commun, précisément, d'être des modes d'expression d'une opération d'identification ${ }^{3}$. Nous verrons que cette opération peut prendre des formes extrêmement variées, dont certaines sont d'ailleurs purement discursives.

Le propos de cet article se veut essentiellement descriptif. L'utilisation qui y sera faite des exemples est délibérément illustrative. On ne cherchera pas à donner des indications quantitatives sur les phénomènes décrits, en menant par exemple une exploration systématique d'un corpus fermé. Les exemples présentés sont «tout venant», écrits aussi bien qu'oraux, et proviennent d'époques variées. Les principaux points abordés seront: l'opération d'identification; les diverses formes que peut prendre le constituant gauche et le rapport qu'il y a entre ces formes et la possibilité ou l'impossibilité de supprimer dans la copule le pronom $c e$; les différents ordres dans lesquels peuvent se présenter les trois constituants de " $\mathrm{A}$ c'est $\mathrm{B}$ »;

2 Martin (1983) utilise l'expression de «focalisation identificatrice» pour décrire les clivées. Pour un examen des différentes formes que peut présenter la copule, voir par exemple van Peteghem (1991), qui décrit la construction qui nous intéresse sous l'appellation de "phrase spécificationnelle».

3 Le terme d' «opération» est important ici. De ce point de vue, l'appellation de "thematic equative» (Halliday, 1985), en raison de l'analogie qu'elle suggère avec les équations, n'est pas très heureuse: dans une équation, le signe '=' n’est pas un opérateur mais un relateur. 
enfin, quelques cas où la distinction entre prédication identificative et prédication attributive est problématique. En revanche, on laissera ici de côté les diverses formulations de la copule et du constituant droit, ainsi que les environnements discursifs dans lesquels interviennent les constructions étudiées ${ }^{4}$.

\section{L'opération d'identification}

En quoi consiste l'opération d'identification marquée par la copule? Un premier point, sur lequel s'arrêtent de nombreux auteurs, est la distinction entre construction identificative et construction attributive. Cependant cette distinction, claire à première vue, se révèle parfois assez difficile à mettre en œuvre sur certaines données. Soient les exemples suivants, présentant tous formellement le même constituant gauche (ce que nous avons $v u$ ):

1) [...] ce que nous avons vu, c'était qu'on creusait de grands trous pour mettre les gens dedans (Ethiopiques 50/51, 1988)

2) [...] Alexandre Adler, franc maçon, sioniste et ancien du CRIF nous dit que ce que nous avons vu, c'était du pipeau. (Forum internet, 2012)

3) Ce que nous avons vu, c'est l'illustration d'une perte de confiance, hélas plus générale, de la part d'une part importante de la population, vis-à-vis de ceux qui nous représentent, nous emploient, et prennent des décisions qui nous concernent. (Article sur: http://www.inspire-institut.org/, 2013)

La copule exprime une prédication identificative dans (1), et attributive dans (2). Dans (1), il s'agit de dire (d'identifier) ce qui a été vu, de sorte que l'énoncé pourrait répondre à la question: Qu'avezvous vu? Dans (2), en revanche, il s'agit de qualifier (caractériser, évaluer, etc.) ce qui a été vu; (2) pourrait donc répondre à des questions comme: Comment jugez-vous ce que vous avez vu?, Que pensez-vous de ce que vous avez vu?, Comment était ce que vous avez vu? On notera que la distinction de ces deux lectures, tout à fait évidente dans (1) et (2), l'est moins dans (3). La présence d'un lexique axiologique

4 Sur ce dernier point ainsi que sur les liens avec la problématique de la phraséologie, voir par exemple Declerck (1988), Roubaud (2000), Legallois et Gréa (2006), Apothéloz (2012; sous presse 2018), Apothéloz et Roubaud (2015). 
(notamment l'expression l'illustration d'une perte de confiance), de même que le côté passablement allusif de la formulation (que désigne au juste l'expression l'illustration d'une perte de confiance?) incite à lire (3) comme attributif. Mais une lecture identificative n'en est pas pour autant exclue. Je reviendrai en fin d'article (section 4) sur quelques cas problématiques analogues.

Les constructions identificatives et attributives impliquent des contextes informationnels différents. Dans les constructions identificatives, le constituant gauche (ici:ce que nous avons vu) est donné comme une expression sémantiquement incomplète. S'agissant de (1), on peut décrire cette incomplétude comme suit: ce que nous avons vu est, dans cet exemple, une expression potentiellement référentielle mais dont l'interprétation référentielle est momentanément suspendue, et donc en attente d'être donnée. La prédication qui suit, marquée par la copule, a précisément pour fonction de donner cette interprétation et ainsi de "résoudre » cette incomplétude. C'est donc, dans cet exemple, une valeur référentielle qui est en jeu dans la prédication identificative. Mais ce type de prédication ne concerne pas toujours la référence.

Je me permets d'insister ici sur cette analyse. Elle revient, de fait, à considérer que le constituant gauche exprime un contenu en attente de complétion. Il y a donc un mécanisme proprement sémiotique qui se joue dans ces constructions. Ce mécanisme peut impliquer, comme dans (1), l'information fournie par le matériau lexical (constituant gauche) et l'interprétation référentielle de cette information (constituant droit), autrement dit la distinction entre «sens» et «référence». À cet égard, la description que donnent des pseudo-clivées les travaux issus de l'école aixoise, consistant à dire que le constituant droit "développe lexicalement» le constituant gauche, ou que la pseudo-clivée «fait passer d'un complément lexicalement non spécifié $[\ldots]$ à un complément lexicalement spécifié» (BlancheBenveniste, 1997: 99) ne me paraît pas rendre compte de ce qui fait la spécificité sémiotique de ces constructions. Ce qui se joue entre les deux constituants, ce n'est pas une spécification "progressive» et purement quantitative du sens lexical, mais la complétude sémantique d'un signe linguistique. Cette double opération a été décrite par certains auteurs comme le fait de poser une variable et de lui attribuer ensuite une valeur (voir par exemple Higgins, 1973; Declerck, 1988). Je reprendrai plus loin cette terminologie. 
Tout autre est le fonctionnement des constructions attributives. Dans l'exemple (2), l'expression ce que nous avons vu est une expression référentielle tout à fait ordinaire et sémantiquement complète: sa référence est donnée comme interprétable, comme connue, et le but de l'énoncé n'est pas de la préciser. La prédication marquée par la copule a dès lors une fonction toute différente: celle de qualifier le référent désigné par l'expression référentielle. Il en va de même dans (3) interprété attributivement.

Indépendamment du phénomène du clivage, l'existence de prédications interprétables comme identificatives ou comme attributives est reconnue depuis longtemps. Damourette \& Pichon (1911-1934, tome 4, \$1546) en donnent un exemple qui est le suivant ${ }^{5}$ :

4) Le chef est un brigand.

Ces auteurs notent que (4) pourrait figurer dans deux types d'environnements:

4a) Une rébellion à main armée a éclaté dans Naples. Le chef est un brigand.

4b) Le chef est un brigand, voilà mon opinion.

Dans le contexte (4a), la formulation (4) pourrait être paraphrasée par: "celui que je définis abstraitement comme chef de la rébellion est un brigand concret déjà connu comme tel». Ces auteurs ajoutent que cette même signification serait également produite par une formulation comme: c'est un brigand qui est le chef. Dans le contexte (4b), la formulation (4) pourrait être paraphrasée par: «l'individu concret que vous connaissez comme étant le chef a les qualités requises pour être qualifié, abstraitement, de brigand». Cette même signification serait également produite par une formulation comme c'est un brigand que le chef.

On peut reformuler cette analyse en disant que dans (4a), le SN défini le chef est sémantiquement incomplet, son interprétation référentielle étant en attente de résolution; le SN indéfini un brigand y est une expression référentielle au sens ordinaire du terme, qui vient résoudre cette incomplétude. La prédication est donc identificative. Dans (4b), en revanche, le chef est une expression référentielle ordinaire, désignant un individu bien identifié; et un brigand est

5 Voir d'autres exemples en anglais discutés par Gundel (1977) ou Lambrecht (1994). 
une expression à lecture purement intensionnelle, visant à qualifier l'individu en question, à porter un jugement sur lui ${ }^{6}$. L'énoncé signifie alors que le locuteur qualifie de «brigand» le chef en question. La prédication est attributive ${ }^{7}$.

Cette ambiguïté peut être exploitée dans divers jeux langagiers. C'est très exactement ce qui se passe dans le passage suivant de L'Avare:

5) LA FLÈCHE: Je dis que la peste soit de l'avarice et des avaricieux. HARPAGON: De qui veux-tu parler?

LA FLÈCHE: Des avaricieux.

HARPAGON: Et qui sont-ils, ces avaricieux?

LA FLÈCHE: Des vilains et des ladres. (Molière, L'Avare, I, 3, 1668)

En demandant Et qui sont-ils, ces avaricieux?, Harpagon attend évidemment une réponse en termes d'identification: il ne fait que reformuler la question De qui veux-tu parler? posée précédemment. Mais La Flèche fait mine de ne pas comprendre, ou de comprendre autre chose, et répond au moyen d'une qualification, c'est-à-dire d'une expression attributive.

Higgins (1973) a comparé les propriétés sémantiques du constituant gauche des constructions identificatives à celles des intitulés de liste. Un intitulé de liste, en effet, ne désigne pas les éléments de la liste; il donne seulement une description générale à laquelle chaque élément de la liste est censé être conforme. Dans le même ordre d'idée, le fonctionnement sémantique de ces mêmes constituants gauches a également été comparé par Blanche-Benveniste (2010) à celui de certains titres de livres, films, tableaux, etc., comme: Ce que je crois (J. de Romilly), Tout ce que vous avez toujours voulu savoir sur le sexe sans jamais oser le demander (W. Allen), Ce que j'ai voulu taire (S. Márai), Celle qui en savait trop (L. Barclay), etc.

6 La distinction entre usage référentiel et usage attributif des syntagmes nominaux remonte à Donnellan (1966) et a donné lieu à diverses discussions et controverses. Sur cette distinction et dans le domaine francophone, voir par exemple Kleiber (1981) et Karolak (1995).

7 Damourette et Pichon (1911-1934, t. 4: 545) utilisent les expressions de «syndèse concrétante» et de "syndèse abstrayante» pour désigner respectivement ces deux types de prédications. Comme le note van Peteghem (1991: 26), cet usage des notions de "concret» et d' "abstrait» renvoie vraisemblablement, chez ces auteurs, au caractère plus ou moins référentiel des expressions apparaissant à gauche et à droite de la copule. 
Notons enfin que quelques rares auteurs (Higgins, 1973; Declerck, 1994) ont utilisé le terme de "pseudo-clivées» pour désigner toutes

les constructions en "A c'est B», qu'elles soient identificatives ou attributives. Ce choix ne fait bien entendu que déplacer le problème et les conduit ensuite à distinguer entre pseudo-clivées identificatives et de pseudo-clivées attributives.

\section{Types d'expressions pouvant fonctionner comme constituant gauche}

Passons en revue les principales formes que peut prendre le constituant gauche. On distinguera les expressions de rang nominal (2.1.), équivalant fonctionnellement à des syntagmes nominaux, et les expressions de rang propositionnel (2.2.). Comme on va le voir, cette distinction trouve une justification supplémentaire dans le fait qu'elle a des conséquences sur la réalisation de la copule.

Les cas suivants seront abordés:

2.1.1. Relatives nominales

2.1.2. SN ayant comme tête un nom ou une forme nominalisée d'un adjectif ou d'un participe

2.2.1. Propositions existentielles ou présentatives

2.2.2. Propositions présentant la forme d'une question

2.2.3. Propositions subordonnées

\subsection{Expressions de rang nominal}

\subsubsection{Relatives nominales}

Il s'agit des expressions constituées d'un pronom démonstratif (ce, celui, celle, etc.) ou d'un adverbe locatif (là, partout) fonctionnant comme un antécédent, sur lequel branche une proposition relative. L'expression de "relative périphrastique» est parfois également employée pour désigner ce type d'expression (Riegel et al., 2009). L'ensemble équivaut à un syntagme nominal.

6) ce que je préfère celui dont ils se méfient ce qui a manqué ce qui s'est passé là où il dort le mieux etc. 
L'expression servant d'antécédent au pronom relatif peut être remplacée par un nom. Il s'agit souvent d'un nom à faible contenu sémantique, comme chose, truc, personne, endroit, moment, etc. Ce nom a parfois été décrit comme une sorte de proforme nominale (Blanche-Benveniste et al., 1990):

7) la chose que je préfère

la personne dont ils se méfient

le truc qui a manqué

la chose qui s'est passée

l'endroit où il dort le mieux

etc.

Le verbe de la relative peut lui aussi être instancié par une proforme verbale (faire), comme dans les énoncés suivants:

8) [...] tout ce que je fais, c'est de prendre une goutte, une larme de cognac pour m'aider à m'endormir. (Simon, L'Herbe, 1958)

9) Ce qu'il faut faire, c'est bien surveiller son chien pendant les heures qui suivent la piqûre. (Forum internet, 2017)

Quand le verbe de la relative comporte un semi-auxiliaire (modal, aspectuel), il arrive que la proforme verbale soit absente.

10) Ce qu'il faut, c'est contenir les migrants avant qu'ils arrivent dans les Alpes-Maritimes. (Homme politique, Nice-Matin, 2017)

11) Ce qu'il devrait, surtout, c'est faire soigner sa vision. (Evanovich, Septième ciel, 2014) $)^{8}$

12) Le gouvernement, ce qu'il aurait dû commencer, c'est d'abord par ne pas faire la guerre. (Calaferte, C'est la guerre, 1993)

En principe, toutes ces formulations peuvent également se présenter sous une forme non segmentée (autrement dit prosodiquement liée), dans laquelle la copule est réduite au seul verbe être, sans le pronom ce. Tel est le cas de l'exemple ci-dessous:

8 Étant entendu, que dans cet exemple, faire n'est pas une proforme mais le verbe faire causatif. Le rétablissement de la proforme donnerait: Ce qu'il devrait faire, surtout, c'est faire soigner sa vision. On observera au passage que faire causatif ne peut pas, contrairement à faire proforme, être détaché, dans une pseudo-clivée, du verbe sur lequel il porte. Dans Ce qu'il devrait faire, c'est soigner sa vision, faire ne peut pas être interprété comme causatif. Dans l'exemple (12), faire a un autre statut encore, celui de verbe support. 
13) Ce qui a ajouté au désarroi de la population est l'adoption, et son entrée immédiate en vigueur, d'une loi sur un impôt supplémentaire destiné à réunir des fonds pour financer l'engagement de l'armée au Kosowo. (Libération, 1998)

Valli (1981) a formulé l'hypothèse selon laquelle l'absence de ce pourrait parfois s'expliquer par des raisons sociolinguistiques et être analysée comme un fait d'hypercorrection.

Dans le modèle syntaxique adopté par Roubaud (2000), seules les constructions identificatives présentant un constituant gauche de type (2.1.1.) sont considérées comme des pseudo-clivées. Le verbe de la relative nominale y est analysé comme le verbe recteur de la construction. La proforme est régie par ce verbe, et la place de rection qu'elle représente est réinstanciée et développée par le constituant droit.

\subsubsection{SN ayant comme tête un nom ou une forme nominalisée d'un adjectif ou d'un participe}

Une forme très fréquente du constituant gauche est un SN dont la tête est un adjectif ou un participe présent nominalisé:

14) [...] l'essentiel c'est qu'il vous encourage. (Echenoz, Les Grandes blondes, 1995)

15) Mais le plus plaisant dans ces universités, c'est souvent le nom qu'elles portent. (Genette, Bardadrac, 2006)

16) Pour beaucoup de réalisateurs, le plus dur c'est de trouver un producteur, pour le producteur de croire au projet et de convaincre les autres partenaires [...]. (Journal du Caméo, 2006)

Une autre forme fréquente est celle d'un SN à tête nominale ${ }^{9}$ :

17) le miracle de ce film// [...] c'est que. c'est un film où ça va mal pour absolument tout $l=$ monde. pratiquement. et où on n'arrête pas. soit de sourire soit de rire franchement $\backslash$ (Radio, interview de Ferreira Barbosa, 1997) ${ }^{10}$

9 Pour des données complémentaires concernant les types d'adjectifs nominalisés, de participes présents nominalisés et de noms apparaissant le plus souvent dans ce type de contexte, voir Roubaud (2000) et Legallois et Gréa (2006). Schmid (2001) apporte également des données intéressantes concernant l'anglais.

10 Conventions de transcription: '//' indique un contour prosodique fortement ascendant, 'I' un contour prosodique fortement descendant, et '.' une très courte pause. 
18) Surtout, notre point de conflit le plus important, c'est qu'on peut pas s'exprimer à l'aise. (Grimm, La Flambe, 1982)

Comme dans la rubrique 2.1.1., ces formulations peuvent également se présenter sous une forme non segmentée (copule sans pronom):

19) L'important est que cela soit dit depuis que l'enfant est tout petit.

(F. Dolto, La Cause des enfants, 1985)

20) Mais le plus étonnant est que des pays comme les États-Unis et le Canada n'en fassent pas partie. (Site de la Heinrich Böll Stiftung, 2017)

21) L'urgence aujourd'hui n'est pas le statut des chaînes commerciales, c'est la situation du service public, victime d'intoxications publicitaires. (Libération, 1998)

On observera que l'extrait (21) comporte deux constituants droits, l'opération d'identification étant associée ici à une mise en opposition (n'est pas/c'est) ${ }^{11}$. Il est intéressant de noter que la copule, dans cet exemple, est réalisée d'abord sans pronom (pour la partie réfutative de l'opposition), et ensuite avec pronom (pour la partie assertive).

Blanche-Benveniste (2010) a montré que l'opération d'identification peut entretenir deux types de rapports avec le nom. Bien que la distinction de ces deux types de rapports ne soit pas toujours facile à établir, et qu'il y ait vraisemblablement des cas de double analyse, on peut distinguer:

- les cas où le constituant droit apporte un "complément» au nom, comme dans les exemples suivants:

22) Mon plaisir, c'est de rester assis encore quelques minutes pendant que le générique défile. (Gavalda, Ceux qui savent comprendront, 2000)

23) Son intérêt, c'était le lieu de la tragédie, et non pas la tragédie elle-même, encore moins son héros. (Labro, Des bateaux dans la nuit, 1982)

On a en effet: mon (le) plaisir de rester assis..., son intérêt pour le lieu de la tragédie, avec un statut de complément déterminatif pour les expressions de rester assis... et pour le lieu de la tragédie;

11 Pour une étude du rôle des pseudo-clivées dans ce type de contexte, voir par exemple Apothéloz (2012) et Apothéloz et Roubaud (2015). 
- les cas où le nom du constituant gauche a une valeur attributive, catégorisante, et où le constituant droit identifie le support de cette attribution, de cette catégorisation:

24) Sa récompense, c'est qu'il lui est permis d'être chrétien et d'obéir. (Barth, Dogmatique, 13, 1989)

25) Le comble, c'est que sa sœur se marie aussi. (Bood, Les Années doubles, 1974)

Dans des exemples comme (24)-(25), mais aussi (19)-(21), le locuteur formule dans un premier temps une prédication attributive, et dans un second temps indique (identifie) le support de cette prédication.

Parmi les énoncés dont le constituant gauche est un nom, on peut mentionner le cas particulier des énoncés définitionnels:

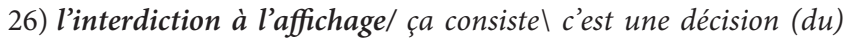
ministère de l'Intérieur. absolument arbitraire et: qui n'est soumise à AUcun jug=ment et qui n'a aucune ne souff= d'aucun appel/ qui consiste à interdirel. la: l'exposition \. publiquel. de: de tout journal euh interdit/ non seul=ment de l'exem-d'un de l'exemplaire visél mais tous les exemplaires PRÉcédents/ et tous les exemplaires. futurs $\backslash$. (Oral, Cavanna, p. 13, corpus F. Zay) ${ }^{12}$

Bien qu'il s'agisse d'une catégorie assez difficile à circonscrire, les énoncés définitionnels mettent en jeu un autre type d'incomplétude que les énoncés proprement identificatifs examinés jusqu'ici. Comme le notent Legallois et Gréa (2006), les définitions répondent à la question qu'est-ce qu'un $N$ ?, tandis que les énoncés identificatifs répondent à la question quel est le N? Par ailleurs, même si leur definiendum (constituant gauche) peut être formulé sous la forme d'un SN défini, et masquer son caractère autonymique, comme dans l'exemple ci-dessus, les définitions présentent une dimension métalinguistique que n'ont pas les constructions identificatives. Leurs deux constituants (definiendum, definiens) opèrent de façon exclusivement intensionnelle et sont donnés comme substituables. On entend évidemment ici par «définition» aussi bien des énoncés de type lexicographique que des

12 Les signes ' $\%$ ' et ' ' notent respectivement un contour prosodique ascendant et descendant; '. note une courte pause; '?' note un allongement de la dernière syllabe; les petites capitales notent une emphase prosodique. 
définitions spontanées telles que nous en produisons quotidiennement, y compris celles qui poursuivent des finalités ironiques, ludiques, etc.

\subsection{Expressions de rang propositionnel}

Je distinguerai ici trois cas: (2.2.1.) celui des propositions existentielles ou présentatives; (2.2.2.) celui des propositions présentant la forme d'une question; (2.2.3.) enfin, celui des propositions subordonnées. L'une des propriétés formelles que partagent les constructions identificatives dont le constituant gauche est de rang propositionnel est que la copule comporte toujours le pronom ce; la construction est donc toujours segmentée. En revanche, les exemples des deux rubriques précédentes (2.1.1. et 2.1.2.) peuvent en principe toujours être formulés sans la présence de ce pronom.

\subsubsection{Propositions existentielles ou présentatives}

La proposition a une forme assertive et comporte un syntagme nominal qui a un statut particulier, en ce sens que c'est lui qui fait l'objet de l'opération d'identification exprimée par le constituant droit. Dans les exemples ci-dessous, une particularité et une chose ont très clairement ce statut: ${ }^{13}$

27) en France nous avons une particularité/ c'est que tout commence et se termine par l'État (Radio, 2005)

28) Une chose m'étonne, marmonna Postel-Wagner, c'est que Fraenkhel ait pu exercer si longtemps en souffrant ce qu'il a souffert. (Pennac, Monsieur Malaussène, 1995)

La valeur existentielle ou présentative est souvent explicitement formulée, typiquement au moyen de l'expression il y $a$ :

29) Les livres sont des amis parfaits. Mais il y a une chose qu'ils ne peuvent souffrir, c'est d'être prêtés. Ils sont alors si vexés qu'ils ne reviennent jamais. (Mot d'auteur, attribué à G. Feydeau)

13 Sur l'interprétation existentielle (vs partitive) des SN indéfinis, voir Kleiber (2001). 


\subsubsection{Propositions présentant la forme d'une question}

Dans l'oral ordinaire, on trouve assez souvent des constructions identificatives dans lesquelles le constituant gauche a la forme d'une question. Roubaud (2000) en donne plusieurs exemples. Ces questions peuvent porter sur le sujet grammatical ou tout type de complément (dans les transcriptions de cette auteure, le signe ' + ' note une pause):

30) qui organise tout cela finalement c'est un certain Léo Burleo (Oral, Roubaud, 2000: 108)

31) qu'est-ce que je faisais c'est que j'allais jouer dehors (Oral, Roubaud, 2000:108)

32) où je vais le plus en somme euh + ces années-ci à Orléans c'est + aux films de Connaissance du Monde (Oral, Roubaud, 2000: 283)

33) pourquoi ils s'affolent c'est que dans son programme Mitterrand envisageait la fermeture des écoles libres (Oral, Roubaud, 2000: 108)

34) comment il l'a su c'est qu'elle lui a donné le numéro (Oral, Roubaud, 2000: 108)

35) quand ils m'ont énervé c'est quand ils m'ont répondu (Oral, Roubaud, 2000: 108)

On notera que le constituant gauche pourrait ici être reformulé, avec une différence sémantique finalement assez minime, selon le schéma traité dans la rubrique 2.1.1.:

30a) qui organise tout cela finalement $\approx$ celui qui organise tout cela finalement

31a) quest-ce que je faisais $\approx$ ce que je faisais

32a) où je vais le plus $\approx$ là où je vais le plus

33a) pourquoi ils s'affolent $\approx$ la raison pour laquelle ils s'affolent

34a) comment il l'a su $\approx$ la façon dont il l'a su

35a) quand ils mont énervé $\approx$ la fois où ils mont énervé.

Les exemples (30)-(35) ne doivent pas être confondus avec le cas des constructions identificatives inversées au sens de Higgins (1973), dans lesquelles le constituant sémantiquement

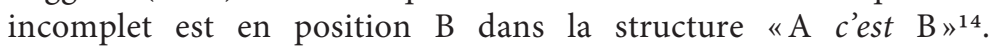

14 Higgins (1973) les qualifie de «inverted», Collins (1991) de «reversed». 
Il en irait ainsi dans les formulations suivantes, forgées à partir des exemples ci-dessus:

36) Qui organise tout cela finalement, c'est ce qu'elle voudrait bien savoir.

Qu'est-ce que je faisais, c'est ce qu'elle voudrait bien savoir.

Où je vais le plus en somme, c'est ce qu'elle voudrait bien savoir.

Pourquoi ils s'affolent, c'est ce qu'elle voudrait bien savoir.

Comment il l'a su, c'est ce qu'elle voudrait bien savoir.

Quand ils m'ont énervé, c'est ce qu'elle voudrait bien savoir.

Ce cas sera examiné plus loin, section 3.

\subsubsection{Propositions subordonnées}

Les constructions concernées dans cette rubrique sont, pour l'essentiel, celles dont le constituant gauche est une subordonnée introduite par les conjonctions quand, lorsque ou $s i$ :

37) Parfois je ne supporte pas mais parfois ça ne me fait rien. Quand je ne supporte pas c'est que je suis de mauvaise humeur. (Akerman, Ma mère rit, 2013)

38) [...] lorsqu'elle rit, c'est par explosion, en montrant toutes ses dents. (Green, Journal, 1934)

39) S'ils ont si peur de la vérité, c'est qu'elle leur est défavorable. (Blocher-Saillens, Témoin des années noires, 1938-1945)

Ces subordonnées servent, dans ce contexte identificatif, à introduire une cause, une finalité, une circonstance ou encore une manière. Dans (37), quand je ne supporte pas sert à annoncer une cause ou une circonstance, celle-ci étant développée dans le constituant droit; dans (38), lorsqu'elle rit sert à annoncer une manière; et dans (39), s'ils ont peur de la vérité sert à annoncer une cause ${ }^{15}$. Il en résulte que ces formulations pourraient être regardées comme des variantes du cas (2.1.2.) et donc être reformulées comme suit:

37a) La raison pour laquelle je ne supporte pas..., La circonstance dans laquelle je ne supporte pas...

38a) La manière/façon dont elle rit...

39a) La raison pour laquelle ils ont peur de la vérité...

15 Sur ces constructions en si... c'est que, voir Corminboeuf (2009), Sabio (2013) et Kuyumcuyan (2017). 
On voit bien ici la différence avec les exemples examinés dans la rubrique (2.2.2.). Dans les exemples du type (2.2.2.), la proposition interrogative exprime une variable qui est sémantiquement circonscrite par l'élément interrogatif (qui, où, comment, quand, etc.), et c'est cette variable qui se voit ensuite attribuer une valeur. Dans les exemples du type (2.2.3.), en revanche, la proposition sert également à exprimer une variable mais de façon en quelque sorte indirecte. Ainsi, dans (37) et (38), une subordonnée temporelle en quand ou lorsque est utilisée pour annoncer et exprimer une cause, une circonstance ou une manière; et dans (39), une subordonnée conditionnelle (en si) est utilisée pour annoncer et exprimer une cause. Les conditionnelles peuvent d'ailleurs aussi être utilisées pour exprimer une finalité (ex. 40) ou une manière (ex. 41). C'est alors cette finalité ou cette manière qui est identifiée par le constituant droit, comme dans les extraits ci-dessous:

40) Je n'ai nullement la pensée de vous témoigner de la méfiance, et je suis bien sûre que ce n'est pas l'intention de mon ami Hetzel. Si je désire agir régulièrement, c'est afin de vous témoigner que je veux me conformer à une clause dont vous m'avez reproché [...] de m'être écartée, en vendant certains ouvrages sans vous offrir la préférence. (Sand, Corresp., 1851)

41) [Il est question de Salluste] S'il pense, c'est toujours du fond des faits qu'il fait sortir à la lumière [...] ; s'il peint, c'est toujours d'après les traits naturels de son Héros, \& avec des couleurs tranchantes; s'il parle, c'est toujours sans art [...]. (Comte d'Albon, Discours sur cette question: Si le Siècle d'Auguste doit être préféré à celui de Louis XIV, relativement aux Lettres et aux Sciences, 1784)

Dans les constructions en si des exemples (39)-(41), la proposition introduite par si perd pratiquement sa valeur conditionnelle et «marque un fait dont la proposition suivante donne la raison» (Sandfeld, 1977: 359-360). Dans son ouvrage sur l'expression de l'hypothèse, Corminboeuf (2009) la qualifie pour cette raison de «factuelle». Selon cet auteur, l'effet de la conjonction est d'associer, dans si-p, la validation de $p$ à une "clé de validation". L'opération d'identification signifiée par la copule a précisément pour effet de fournir cette clé, en associant à $p$ une circonstance, une cause efficiente ou finale, une 
manière (cf. Corminboeuf, 2009: 296, 336) ${ }^{16}$. La proposition $p$ est validée dans les cas où cette circonstance, cause ou manière est réalisée. Ce fonctionnement montre bien la parenté, par ailleurs repérée depuis longtemps (par ex. Martin, 1983), entre les questions totales et la conjonction si.

Il est intéressant d'observer qu'il y a, dans les formulations (37)(41), un décalage entre le sens du constituant gauche (où s'exprime littéralement une temporalité ou une condition) et celui du constituant droit (où s'exprime une cause efficiente ou finale, une circonstance, une manière). En d'autres termes, le constituant gauche, par une sorte de métonymie, introduit de fait une autre variable (cause, circonstance, manière) que celle qu'il signifie littéralement. C'est précisément cet élément qui est sémantiquement incomplet dans le segment gauche, et qui fait l'objet de l'opération d'identification. Ce décalage peut rendre le repérage de l'opération d'identification plus difficile. Néanmoins, les reformulations sémantiquement proches (37a)-(39a), ainsi que celles, analogues, qu'on pourrait produire à partir de (40)-(41), constituent un argument pour considérer qu'on est bien en présence de constructions identificatives.

Comme dans la rubrique (2.2.2.), il y a ici aussi des confusions possibles avec des formes proches. Ainsi, dans l'exemple suivant, la subordonnée en si est une proposition interrogative, et la construction identificative est inversée, le constituant sémantiquement incomplet étant en position B.

42) S'il pense, c'est ce que je voudrais bien savoir. (ex. forgé)

La subordonnée de (42) est donc "percontative» et non pas «intégrative» - pour reprendre la terminologie introduite par Damourette et Pichon (1911-1934: \$1247) ${ }^{17}$.

16 Dans le même ordre d'idée, et pour expliquer la posture épistémique particulière qui s'exprime dans ces si-p, Kuyumcuyan (2017: 56) voit dans $p$ une "coloration» de mention. Cet effet sémantique peut aboutir à une interprétation dialogique des séquences si-p, c'est que $q$, dans laquelle $p$ et $q$ renvoient à des sources distinctes. Sur les conditionnelles et la polyphonie, voir également Achard-Bayle (2013).

17 Pour une analyse de constructions comme (42) mais dans leur arrangement standard (non inversé), voir Roubaud et Sabio (2010). 
La subordonnée peut également servir à introduire un syntagme nominal sémantiquement incomplet. C'est alors ce dernier qui est résolu par le constituant droit:

43) Quand il offre quelque chose, c'est toujours des fleurs. (ex. forgé)

44) Mais s'il y a une chose que je regrette, c'est bien de n'avoir jamais osé essayer, même d'une manière ridicule, de voir ce que c'est de toucher un instrument. (Pérec, Entretiens et conf. II, 1979-1981)

45) Mais, s'il y a une race suspecte, c'est celle des taverniers et des hôteliers. (Faral, La vie quotidienne au temps de saint Louis, 1942)

L'opération d'identification porte ici respectivement sur quelque chose (ex. 43), une chose (ex. 44) et une race suspecte (ex. 45). Ces exemples sont proches des propositions existentielles ou présentatives décrites dans la rubrique (2.2.1.).

\section{Ordre des constituants}

Les trois constituants des constructions identificatives ne se présentent pas toujours dans l'ordre « $\mathrm{A}$ c'est $\mathrm{B}$ » indiqué au début de cet article - ordre considéré en général, implicitement ou explicitement, comme canonique. La copule ne pouvant en principe pas se trouver en dernière position, quatre arrangements sont théoriquement possibles, selon que (1) A précède ou suit $B$, et (2) la copule se trouve entre les deux constituants ou en tête d'énoncé. Pour les décrire, il est utile de se doter d'appellations désignant non pas, comme jusqu'ici, la position des constituants mais leur fonction.

\subsection{Variable, valeur et dynamique informationnelle}

Pour désigner respectivement le constituant sémantiquement incomplet (plus exactement, le constituant comportant un élément donné comme sémantiquement incomplet) et le constituant qui le complète, je reprendrai les deux termes utilisés par Declerck (1988) de variable et de valeur (comme j'ai déjà eu l'occasion de le faire plus haut). Je noterai désormais ces termes en petites capitales, pour les distinguer d'autres usages que je pourrais faire par ailleurs de ces mêmes termes. 
Les quatre arrangements se caractérisent par des dynamiques informationnelles différentes. Envisagés du point de vue de la réalité orale, ils peuvent bien sûr se prêter à diverses réalisations prosodiques, susceptibles elles-mêmes de modifier ces dynamiques. Les principaux points à prendre en considération pour décrire ces dynamiques sont les suivants:

- le statut des constituants VARIABLe et VAleur (thématique, prédicatif ou post-prédicatif),

- la place de la copule prédicative (initiale ou non),

- les propriétés présuppositionnelles du constituant VARIABLE.

On reconnaît généralement que le constituant VARIABLE des constructions identificatives (le constituant gauche, dans la forme canonique examinée jusqu'ici) est porteur d'un présupposé d'existence (par ex. Levinson, 1983: 182-183). Ainsi, l'expression ce que je préférerais comporte un présupposé paraphrasable par «il y a quelque chose que je préférerais». Cette propriété vaut pour toutes les constructions identificatives, à une exception cependant: quand le constituant VARIABLE est lui-même une proposition présentative ou existentielle, comme dans les exemples (27)-(28) répétés ci-dessous. Dans ce cas, l'existence n'est pas présupposée mais ouvertement assertée:

27) en France nous avons une particularité/ c'est que tout commence et se termine par l'État (Radio, 2005)

28) Une chose métonne, marmonna Postel-Wagner, c'est que Fraenkhel ait pu exercer si longtemps en souffrant ce qu'il a souffert. (Pennac, Monsieur Malaussène, 1995)

Or, l'un des rendements discursifs de la présupposition est de permettre de proroger sur le mode de l'implicite une information antérieurement donnée. Autrement dit, la présupposition est un mode d'expression de l'anaphore. Cela explique que, dans les constructions identificatives, le constituant VARIABLE soit très souvent une reprise d'une information antérieure, comme dans (46), ou désigne une information inférable d'une information antérieure, comme dans (47), selon la logique de l'anaphore associative. Dans ce dernier exemple, l'expression ce qu'il sait encore renvoie associativement au contenu sémantique évoqué par les expressions verbales il a su et il a oublié:

46) Par contre, il semble qu'il reste une différence quand on fait intervenir des contenus et la différence ce n'est peut-être pas 
tellement de savoir si les choses sont perceptibles ou non [...]. (Langage \& Travail 1)

47) Aujourd'hui, Godard a oublié comment on fait une image. Il a su, il a oublié. Ce qu'il sait encore, c'est qu'il ne sait plus. Redevenu meilleur critique que cinéaste, il tire plus vite que tout le monde sur ses propres films. (Libération, 1999)

Si on note par TH et PR les constituants thématique et prédicatif, les quatre arrangements possibles peuvent être décrits selon le tableau 1 :

Tableau 1. - Quatre arrangements des constructions identificatives

\begin{tabular}{|c|l|l|}
\hline TYPE & \multicolumn{1}{|c|}{ ORDRE DES CONSTITUANTS } & \multicolumn{1}{c|}{ EXEMPLE } \\
\hline Type 1 & {$\left[{ }_{\text {VARIABLE }}\right]_{T H}-[\text { copule }- \text { VALEUR }]_{P R}$} & ce que je préférerais c'est qu'on sorte tout de suite \\
\hline Type 2 & {$[\text { VALEUR }]_{T H}-[\text { copule }- \text { VARIABLE }]_{P R}$} & qu'on sorte tout de suite c'est ce que je préférerais \\
\hline Type 3 & {$[\text { copule }- \text { VARIABLE }]_{P R}-[\text { VALEUR }]_{T H}$} & c'est ce que je préférerais qu'on sorte tout de suite \\
\hline Type 4 & {$\left[\right.$ copule $-V_{\text {VALEUR }]_{P R}}-[\text { VARIABLE }]_{T H}$} & c'est qu'on sorte tout de suite (ce) que je préférerais \\
\hline
\end{tabular}

Deux raisons au moins amènent à considérer le Type 1 comme davantage «canonique» que les autres ${ }^{18}$.

En premier lieu, on peut considérer que l'ordre consistant à énoncer le constituant thématique avant de prédiquer sur lui est davantage coopératif. En effet, cet arrangement assure une progression de l'information dont le traitement (notamment le traitement interprétatif) est moins coûteux que l'ordre inverse. Quand c'est le constituant prédicatif qui est formulé en premier, comme dans les Types 3 et 4, le locuteur prédique en quelque sorte «à crédit» (Berrendonner, 2012), engendrant ainsi une attente et un coût mémoriel particuliers.

En second lieu, dans la mesure où les constructions identificatives consistent à attribuer une VALEUR à une VARIABLE, on peut, selon la même logique, considérer qu'il est plus coopératif de formuler le constituant exprimant la VARIABLE avant celui exprimant la VALEUR.

Si ce raisonnement est correct, il n'est donc pas surprenant que l'ordre actualisé par le Type 1 soit l'ordre préféré. Toutes choses égales par ailleurs, un locuteur idéalement coopératif appliquera donc les deux principes suivants: (a) placer le thème avant la prédication;

18 Collins (1991) utilise l'expression de «basic pseudo-clefts» pour désigner les pseudo-clivées de Type 1. 
(b) faire correspondre VARIABLE et thème, d'une part, et VALEuR et prédication, d'autre part. On observe alors que:

- le Type 2 transgresse le principe (b),

- le Type 3 transgresse les principes (a) et (b),

- le Type 4 transgresse le principe (a).

Il convient de préciser que le terme de transgression ne doit pas être interprété ici dans un sens normatif. Les Types 2, 3 ou 4 sont en général justifiés par le contexte informationnel dans lequel la construction est utilisée. Le choix d'un type relève essentiellement d'une logique d'accommodation au contexte informationnel.

Le Type 2 correspond aux pseudo-clivées «inversées» (Higgins, 1973). Il conserve l'ordre thème-prédicat, mais inverse VARIABLE et VALEUR.

Les Types 3 et 4 ont en commun de placer le constituant prédicatif en première position, ce qui engendre un effet de focalisation. On pourrait appliquer à ces deux constructions ce que Berrendonner écrit à propos de certaines routines discursives:

Le locuteur met, si lon peut dire, la charrue avant les bœufs: au lieu de poser d’abord, pour prédiquer ensuite, il prédique d'abord «à crédit» sur un objet non identifié, qu'il pose seulement ensuite. (Berrendonner, 2012: 190)

L'effet n'est toutefois pas le même pour le Type 3 et pour le Type 4 . Dans le Type 4, qui correspond aux constructions dites "clivées" (it-clefts), le constituant initial est habituellement marqué, à l'oral, par une emphase prosodique, tandis que le constituant final est énoncé avec un contour prosodique d'appendice (au sens de Mertens, 1990) signalant une fonction de rappel ou de confirmation d'un thème en cours de traitement. Dans le cas du Type 3 (le moins coopératif), le constituant final a un statut différent, dans la mesure où c'est le constituant valeur qui est mis en position post-prédicative, constituant dont la fonction canonique est justement d'être prédicatif. La fonction de ce constituant semble plutôt relever de la réparation que du simple rappel. Cela dit, il est évident que seule une exploration systématique et d'une certaine ampleur pourrait nous éclairer sur les contextes d'utilisation de ces trois variantes de constructions et donc sur leur dynamique informationnelle. 


\subsection{Exemples des Types 2, 3 et 4}

Examinons quelques exemples attestés des Types 2, 3 et 4 . Chaque exemple est suivi de sa version canonique (Type 1) signalée par le signe ' $\rightarrow$ '. La copule et le constituant VARIABLE sont chaque fois en gras.

3.2.1. Type 2: $[\text { VALEUR }]_{\mathrm{TH}}-[\text { copule - VARIABLE }]_{\mathrm{PR}}$

En voici deux illustrations:

48) À quel point il appartient au président de gêner ou de faciliter une déposition (fût-ce inconsciemment), c'est ce que je sens de nouveau, non sans angoisse, et combien il est malaisé pour le juré de se faire une opinion propre, de ne pas épouser celle du président. (Gide, Souvenirs de la cour d'assises, 1914)

$\rightarrow$ Ce que je sens de nouveau, non sans angoisse, c'est à quel point il appartient au président de gêner ou de faciliter une déposition (fût-ce inconsciemment), et combien il est malaisé pour le juré de se faire une opinion propre, de ne pas épouser celle du président.

49) Que ce genre de vie ait de profondes racines, qu'il tienne au sol autant qu'à des habitudes, c'est ce que montre sa persistance, sa généralité dans les contrées auxquelles il s'est adapté; (Vidal de la Blache, Principes de géographie humaine, 1921)

$\rightarrow$ Ce que montre sa persistance, sa généralité dans les contrées auxquelles il s'est adapté, c'est que ce genre de vie a de profondes racines, qu'il tient au sol autant qu'à des habitudes.

On observera que dans l'extrait (48), le constituant VARIABLE est donné de façon discontinue, en deux séquences: la première précède le

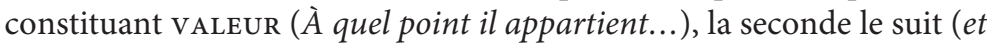
combien il est malaisé...).

\subsubsection{Type 3 : $[\text { copule }- \text { VARIABLE }]_{P R}-[\text { VALEUR }]_{T H}$}

50) D'ailleurs, c'est le vice de la plupart des gens de communauté de croire qu'ils ne peuvent faire de mal en défendant l'honneur de leur corps: cet honneur est une espèce d'idole à qui ils se croient permis de sacrifier tout, justice, raison, vérité. (Racine, Abrégé de l'histoire de Port-Royal, c.a. 1690)

$\rightarrow$ Le vice de la plupart des gens de communauté, c'est de croire qu'ils ne peuvent faire de mal en défendant l'honneur de leur corps. 
51) Et c'est aussi ce qui forme le bonheur des personnes de grande condition qu'ils ont un nombre de personnes qui les divertissent et qu'ils ont le pouvoir de se maintenir en cet état. (Pascal, Pensées, ca. 1650)

$\rightarrow$ Ce qui forme le bonheur des personnes de grande condition, c'est aussi qu'ils ont un nombre de personnes qui les divertissent et qu'ils ont le pouvoir de se maintenir en cet état.

L'exemple oral suivant, emprunté à Müller (2006), est intéressant dans la mesure où il revient, suite à un incident de la formulation, à produire une sorte d'hybride des Types 1 et 3 . En termes de praxéologie langagière, on pourrait en donner la description suivante: le locuteur commence par amorcer une construction de Type 3 (copule en première position); puis, après avoir achevé la formulation du constituant VARIABLE, il poursuit comme s'il se trouvait au milieu d'une construction de Type 1, ce qui se manifeste par la répétition de la copule:

52) c'est un des côtés intéressants de votre séminaire commun [...] c'est que je trouvais que ça ouvrait la porte à ça (Oral, Müller, 2006: 388)

$\rightarrow$ un des côtés intéressants de votre séminaire commun [...] c'est que je trouvais que ça ouvrait la porte à ça

Cet incident de la formulation produit rétroactivement une réinterprétation formelle du constituant initial, réinterprétation dans laquelle la copule initiale est absente ${ }^{19}$. Ici, on peut supposer que c'est parce que son amorce conduisait à une impasse que le locuteur a choisi une autre formulation. La formulation (52a) est certes possible, mais ne comporte pas la modalité qu'exprime je trouvais:

52a) c'est un des côtés intéressants de votre séminaire commun que d'ouvrir / d'avoir ouvert la porte à ça

Cette modalité ne fait d'ailleurs pas partie des éléments impliqués par l'opération d'identification, car c'est une insertion parenthétique. On peut donc supposer que c'est sa présence qui est à l'origine de ce bafouillage.

19 Sur ces changements inopinés de programme pouvant affecter la formulation, voir Apothéloz et Zay (1999). 
3.2.3. Type 4: $[\text { copule - VALEUR }]_{P R}-\left[\right.$ VVARIABLE $_{T H}$

Comme indiqué plus haut, ce type coïncide avec les constructions clivées :

53) C'est arriver à l'heure qui compte pour nous les usagers des TPG! (Forum internet, 2016, TPG = Transports publics genevois)

$\rightarrow$ Ce qui compte pour nous les usagers des TPG, c'est arriver à l'heure.

54) [...] c'est quand il pleuvait que j'allais voir les copains à SaintAndré-Avellin. (http://carnets.contemporain.info/moyens/ archives/294, 2012, 02/01/2018)

$\rightarrow$ quand/le moment où j'allais voir les copains à Saint-AndréAvellin, c'est quand il pleuvait.

\section{Retour sur la distinction « identificatif » vs « attributif»}

Dans leur article sur les constructions identificatives ${ }^{20}$, Legallois et Gréa (2006) ont montré que ces constructions présentent certaines propriétés qui relèvent de la phraséologie. Ils formulent par ailleurs l'hypothèse suivant laquelle le rapport entre constructions attributives et identificatives ne relève peut-être pas de l'homonymie, mais d'un schéma général (une "construction", au sens cette fois-ci des grammaires du même nom) subsumant les deux lectures et attestant de la prégnance d'une même Gestalt syntaxique.

Certains exemples de mon corpus témoignent en effet d'un amalgame entre les deux constructions, entre les deux lectures. Cela concerne tout particulièrement le Type 1 (canonique). Cet amalgame peut prendre la forme d'une sorte de figure grammaticale, typiquement d'une formulation qui se donne toutes les apparences d'une construction identificative mais qui, compte tenu du contexte, doit être interprétée comme attributive. C'est ce qu'on observe dans le texte suivant, où tout lecteur un tant soit peu attentif reconnaîtra un procédé relativement courant dans le genre narratif, notamment quand

20 L'article porte plus particulièrement sur les constructions identificatives dont le constituant gauche est un nom ou une forme nominalisée d'un adjectif ou d'un participe. Ces constructions sont appelées par les auteurs «spécificationnelles». 
il s'agit d'exprimer un constat de changement, en particulier la surprise d'un personnage devant un changement:

55) Antoine ne dit plus rien, parce qu'il le voit. Antoine se tait toujours, regardant fixement devant lui. Ce qu'il voit c'est un homme déjà vieux, sec, avec des petits yeux clairs enfoncés dans des orbites sans sourcils. (Ramuz, Derborence, 1934)

Dans cet extrait, le personnage nommé Antoine voit quelqu'un qu'il connaît de longue date et qu'il reconnaît, en dépit de certaines transformations. Dans le passage en gras, il s'agit donc moins de dire ce que voit Antoine, que comment est ce qu'il voit. La description que donne ici le SN indéfini un homme déjà vieux... a donc un statut à la fois identificatif et attributif. Identificatif, dans la mesure où la personne observée "ne se ressemble plus» au point d'être vue comme une autre personne, dont on ne fait que donner une brève description; attributif, dans la mesure où le personnage nommé Antoine connaît et reconnaît néanmoins cette personne.

Ce phénomène se rencontre en particulier quand le constituant gauche (VARIABLE) comporte un verbe de perception ou assimilé (voir, avoir devant soi, trouver, retrouver...) et que le constituant droit est un $\mathrm{SN}$ indéfini (l'affinité de l'indéfini avec la fonction attributive est bien connue). L'énoncé sert alors à suggérer que la façon dont apparaît le référent est inhabituelle et/ou que celui-ci a subi un changement plus ou moins inattendu. L'exemple (3) discuté au début de cet article, où l'on retrouve le verbe voir dans le constituant gauche, est de ce type ${ }^{21}$.

Voici trois autres illustrations de cet emploi de notre construction:

56) [Un soldatracontecommentilaportésecoursà un enfant, en dépit du fait que celui-ci soit soupçonné d'actes de terrorisme] Tout ce que je voyais, c'était un enfant qui ressemblait à un enfant que je connaissais. (http://www.lapresse.ca/actualites/ national/, juillet 2017)

57) Pour l'étranger, cela faisait un détour, mais il voulait, de làhaut, embrasser le pays, la plaine étalée, et dans les éclaircies des arbres, les toits de chaume, les taches irrégulières des champs et des jardins. Sa face se durcit, plaquée de sueur. Ce qu'il voyait, c'était une étendue torréfiée, d'une sale couleur rouillée, nulle

21 Il serait intéressant de faire le tour des verbes et expressions verbales qui «tirent» la construction du côté attributif, ou qui favorisent la figure dont il est question ici. 
part, la fraîcheur verte qu'il espérait, et çà et là, la moisissure éparse des cases. (Roumain, Gouverneurs de la rosée, 1944)

58) J'attendais le regard vif et impénétrable qui me faisait fondre autrefois, et tout ce que je retrouvais, c'était un oeil vitreux et faible qui me donnait des frissons. (Nayraa, Jusqu'à la mort, 2010)

On est ici en présence d'une variante du schéma phraséologique général auquel font allusion Legallois et Gréa (2006). Il serait évidemment intéressant de documenter cette variante, en fouillant des corpus à partir d'un schéma comme le suivant:

ce que $+\mathrm{V}$ de perception + copule $+\mathrm{SN}$ indéf.

Quoi qu'il en soit, il est légitime, me semble-t-il, de décrire ces formulations comme des figures grammaticales, ainsi que je l'ai fait plus haut: "figures", parce qu'il y a bien une sorte de double jeu, la prédication attributive étant travestie sous la forme d'une prédication identificative; "grammaticale», dans la mesure où les catégories impliquées par ce procédé sont proprement grammaticales (identification vs attribution). Des cas comme (55)-(58) constituent ainsi des données intéressantes en faveur de l'hypothèse formulée par les auteurs précités.

À ces exemples, il convient d'ajouter le cas où la VALEUR prédiquée par la construction identificative est elle-même une attribution. Exprimée ainsi, la chose peut paraître paradoxale, compte tenu de ce qui a été écrit plus haut. Pourtant, rien ne s'oppose à ce qu'un verbe attributif (typiquement, être) figure dans une relative nominale, et que ce soit son attribut qui est mis en suspens, puis résolu dans le constituant droit. L'incomplétude du constituant gauche est alors purement intensionnelle. C'est ce qui se passe dans l'exemple suivant:

59) Papillon, j'ai lu ses Mémoires, ce qu'il est, c'est un menteur, purement et simplement un menteur! (Perrut, Patria o muerte, 2009)

\section{Conclusion}

Partant d'un schéma général « $\mathrm{A}$ c'est $\mathrm{B}$ », dans lequel la prédication «c'est $\mathrm{B}$ » a pour fonction d'affecter une certaine valeur à une variable contenue dans A ou exprimée par A, j’ai examiné les points suivants: 
1) la nature de la relation entre $A$ et $B$, principalement la distinction entre prédication attributive et prédication identificative, ainsi que l'existence de cas ambigus;

2) les différentes formes que peut prendre A: expressions de rang nominal ou expressions de rang propositionnel;

3) les variations que peut subir l'ordre des constituants. Quatre arrangements ont été distingués et exemplifiés. Ces arrangements se caractérisent par des dynamiques informationnelles distinctes, et sont donc susceptibles d'apparaître dans des contextes différents;

4) la fragilité de la distinction entre identification et attribution, et les figures grammaticales auxquelles cette distinction peut donner lieu (cas dans lesquels l'expression simule une opération d'identification pour signifier une attribution).

$\mathrm{Au}$ terme de cet examen, on constate que les constructions identificatives présentent une grande diversité de formes. Plutôt que de constructions, peut-être faudrait-il parler plus généralement d'expressions de l'opération d'identification. Cela est d'autant plus vrai que cette opération peut également être exprimée par des moyens purement discursifs ou conversationnels, ne relevant ni de la micro- ni de la macro-syntaxe. Pour des raisons de place, ces cas n'ont pas été traités dans le présent article. Au sein de cette diversité, les pseudoclivées au sens habituel du terme apparaissent, au fond, comme un cas tout à fait particulier.

\section{Bibliographie}

ACHARD-BAYLE G. (2013), De si à l'imparfait, l'hypothèse polyphonique: modus et dictum, fiction et diction, Cahiers Chronos 26, 39-57.

AKMAJIAN A. (1970), Aspects of the grammar of focus in English, New York, Garland.

APOTHÉLOZ D. (2012), Pseudo-clivées et constructions apparentées, in Groupe de Fribourg (éds), Grammaire de la période, Berne, Peter Lang, 207-232.

APOTHÉLOZ D. (sous presse 2018), Deux exemples de noyaux potentiellement phraséologiques: les pseudo-clivées et "déjà » justificatif, Verbum 40: 1 . 
APOTHÉLOZ D. \& ROUBAUD M.-N. (2015), Constructions pseudoclivées, Encyclopédie Grammaticale du Français, <http:// encyclogram.fr/util/liste.php>.

APOTHÉLOZ D. \& ZAY F. (1999), Incidents de la programmation syntagmatique: reformulations micro- et macro-syntaxiques, Cahiers de linguistique française 21, 11-34.

BERRENDONNER A. (2012), Structures préparation +action, in Groupe de Fribourg, Grammaire de la période, Berne, Peter Lang, 187-205.

BLANCHE-BENVENISTE C. (1997), Approches de la langue parlée en français, Paris, Ophrys.

BLANCHE-BENVENISTE C. (2010), Les pseudo-clivées et l'effet deux points, in Béguelin M.-J., Avanzi M. \& Corminboeuf G. (éds), La parataxe. Structures, marquages et exploitations discursives, tome 2, Berne, Peter Lang, 185-217.

BLANCHE-BENVENISTE C., BILGER M., ROUGET C., VAN DEN EYNDE K. \& MERTENS P. (1990), Le français parlé. Études grammaticales, Paris, Éditions du CNRS.

COLLINS P.C. (1991), Cleft and pseudo-cleft constructions in English, London and New York, Routledge.

CORMINBOEUF G. (2009), L'expression de l'hypothèse en français. Entre hypotaxe et parataxe, Bruxelles, De Boeck-Duculot.

DAMOURETTE J. \& PICHON E. (1911-1934). Des mots à la pensée. Essai de grammaire de la langue française, t. 4, Paris, D'Artrey.

DECLERCK R. (1988), Studies in copular sentences, clefts and pseudoclefts, Leuven, Leuven University Press.

DECLERCK R. (1994), The taxonomy and interpretation of clefts and pseudo-clefts, Lingua 93, 183-220. Review article de Collins (1991).

DONNELLAN K.S. (1966), Reference and definite descriptions, The Philosophical Review 75:3, 281-304.

GROUPE DE FRIBOURG (2012), Grammaire de la période, Berne, Peter Lang.

GUNDEL J. K. (1977), Where do cleft sentences come from? Language $53: 3,543-559$.

HALLIDAY M.A.K. (1985), An introduction to functional grammar, Londres, Edward Arnold.

HIGGINS F. R. (1973), The pseudo-cleft construction in English, M.I.T. dissertation. Bloomington (IN), Indiana University Linguistics Club, 1976. 
KAROLAK S. (1995), Distinction: usage référentiel/usage attributif, valeur spécifique/ valeur non spécifique du syntagme nominal, in Karolak S. (recueil d'articles de cet auteur), Études sur l'article et la détermination, Kraków, Wydawnictwo Naukowe WSP, 207-236.

KLEIBER G. (1981), Problèmes de référence: descriptions définies et noms propres, Université de Metz, Centre d'Analyse Syntaxique.

KLEIBER G. (2001), Indéfinis: lecture existentielle et lecture partitive, in Kleiber G., Laca B. \& Tasmowski L. (éds), Typologie des groupes nominaux, Rennes, Presses universitaires de Rennes, 47-97.

KUYUMCUYAN A. (2017), Aspects linguistiques et pragmatiques de la mise en relief dans les pseudo-clivées en si, in Bilger M., Buscail L. \& Mignon F. (sous la dir. de), Langue française mise en relief. Aspects grammaticaux et discursifs, Perpignan, Presses Universitaires de Perpignan, 49-60.

LAMBRECHT K. (1994), Information Structure and Sentence Form, Cambridge, Cambridge University Press.

LEGALLOIS D. \& GRÉA P. (2006), L'objectif de cet article est de... Construction spécificationnelle et grammaire phraséologique, Cahiers de praxématique 46, 161-181.

LEVINSON S.C. (1983), Pragmatics, Cambridge, Cambridge University Press.

MARTIN R. (1983), Pour une logique du sens, Paris, Presses Universitaires de France.

MERTENS P. (1990), Intonation, Chapitre 4 de: Blanche-Benveniste C., Bilger M., Rouget C., van den Eynde K. \& Mertens P., Le français parlé. Études grammaticales, Paris, Éditions du CNRS, 159-176.

MÜLlER G. (2006), La pseudo-clivée: étude en linguistique interactionnelle, thèse de doctorat, Université de Lausanne, Faculté des lettres.

RIEGEL M., PELLAT J.-C., RIOUL R. (20094), Grammaire méthodique $d u$ français, Paris, Presses Universitaires de France.

ROUBAUD M.-N. (2000), Les constructions pseudo-clivées en français contemporain, Paris, Champion.

ROUBAUD M.-N. \& SABIO F. (2010), Les Si-Constructions et la fonction sujet en français contemporain, Actes du CMLF 2010, http://www.linguistique francaise.org, 2161-2172.

SABIO F. (2013), Les séquences en Si dans les corpus oraux, in Debaisieux J.-M. (éd.), Analyses linguistiques sur corpus: 
subordination et insubordination en français, Paris, Lavoisier, 317-362.

SANDFELD K. $\left(1977^{3}\right)$, Syntaxe du français contemporain. Les propositions subordonnées, Genève, Droz.

SCHMID H.-J. (2001), 'Presupposition can be a bluff: How abstract nouns can be used as presupposition triggers, Journal of Pragmatics 33, 1529-1552.

VALLI A. (1981), Note sur les constructions dites «pseudo-clivées» en français, Recherches sur le français parlé 3, 195-211.

VAN PETEGHEM M. (1991), Les phrases copulatives dans les langues romanes, Wilhelmsfeld, Gottfried Egert Verlag. 